\title{
Efeitos da radiação ionizante sobre a polidpsia induzida por esquema de reforçamento ${ }^{1}$
}

\author{
Alessandra de Moura Brandão \\ Lincoln da Silva Gimenes \\ Universidade de Brasília \\ Júlio Luiz Rodrigues \\ Centro de Estudos - Hospital Santa Lúcia
}

\begin{abstract}
Resumo
O objetivo do presente trabalho consistiu em explorar e avaliar os efeitos da radiação ionizante sobre a polidipsia induzida por esquema de reforçamento. Cinco ratos albinos foram utilizados como sujeitos e divididos em dois grupos: dois sujeitos foram expostos a um esquema de liberação de alimento em tempo fixo de 60 segundos (FT $60 \mathrm{~s}$ ) e três foram expostos a um esquema de reforçamento em intervalo fixo de 60 segundos (FI 60 s). Depois de estabelecida a polidipsia, quatro foram expostos a uma dose de radiação ionizante de 4,5 Gy. Uma segunda exposição à radiação ionizante de 6,0 Gy foi realizada para três sujeitos trinta e seis dias após a primeira exposição. Os resultados demonstraram que, para a dose de 4,5 Gy, tanto a resposta de pressão à barra quanto a polidipsia sofreram decréscimo na sessão realizada 2 horas após a exposição. Para a dose de 6,0 Gy houve uma intensificação dos efeitos da radiação ionizante sobre a resposta de pressão à barra e sobre a polidipsia na sessão realizada 48 horas após a exposição. Os sujeitos sob esquema de reforçamento FI $60 \mathrm{~s}$ sofreram maiores decréscimos em ambas as medidas que aqueles sob esquema FT $60 \mathrm{~s}$. Os dados obtidos replicam dados existentes na literatura, em relação a comportamentos operantes, e estendem para comportamentos adjuntivos os efeitos da radiação ionizante. Os resultados sugerem ainda a interação entre o comportamento operante e o comportamento induzido modulando os efeitos da radiação ionizante.
\end{abstract}

Palavras-chave: Radiação ionizante; comportamento adjuntivo; polidipsia.

\section{Abstract \\ Effects of ionizing radiation on schedule-induced polydipsia}

The aim of the present work was to explore and evaluate the effects of ionizing radiation on scheduleinduced polydipsia. Five food deprived rats were used as subjects and submitted to baseline procedure, after that they were divided in two experimental groups: two subjects were exposed to a fixed-time 60 seconds of food schedule (FT 60 s) and three were exposed to a fixed-interval 60 seconds of food schedule (FI $60 \mathrm{~s}$ ). Once schedule-induced polydipsia was developed, four subjects were exposed to ionizing radiation of 4,5 Gy. A second exposure to ionizing radiation of 6,0 Gy was done with three subjects thirty-six days after the first exposure. For the 4,5 Gy the results showed that even the lever press response or the polidipsia decreased at the session occurred two hours after exposure, with subsequent recovery to baseline levels for three of four irradiated subjects. For the 6,0 Gy the results indicates an intensification of radiation effects upon the lever press response and the polydipsia at the session occurred 48 hours after exposure. Besides, the subjects on fixed interval schedule (FI $60 \mathrm{~s}$ ) showed greater decrements on both measures than that on fixed time schedule (FT $60 \mathrm{~s}$ ). The results of the present experiment reproduce the operant behavior literature data, and extend the effects of ionizing radiation to adjunctive behaviors. These results also suggest the occurrence of an interaction between the operant and the adjunctive behavior modulating the effects of ionizing radiation.

Keywords: Ionizing radiation; schedule-induced polydipsia.

Os primeiros relatos sobre certos padrões de comportamento que ocorrem conjuntamente às respostas controladas pelo esquema de reforçamento foram feitos por Falk (1961a). Em seu experimento com ratos no qual as pressões à barra eram reforçadas com pelotas de alimento segundo um esquema de intervalo variável de 60 segundos (VI 60 s), Falk observou um consumo excessivo de água (polidipsia) durante a sessão experimental sem que os sujeitos estivessem submetidos a qualquer privação da mesma. A polidipsia ocorria principalmente após o consumo da pelota de ração e mantinha-se por toda a sessão que tinha a duração de aproximadamente três horas. Para alguns sujeitos o consumo de água durante a sessão excedeu a $2 / 3$ de seu peso corporal e mostrou-se superior ao consumo de água na gaiola-viveiro nos períodos entre as sessões experimentais. A polidipsia também pode ser observada quando se utilizou esquema de liberação de alimento não contingente à resposta com duração fixa de 60 segundos (FT 60 s), mas não sob esquema de reforçamento contínuo (CRF) (Falk, 1961b). Falk denominou o fenômeno de polidipsia psicogênica, sugerindo que sua natureza seria comportamental e não fisiológica. 
Falk (1971) define o comportamento adjuntivo como um comportamento que é mantido indiretamente pelas variáveis controlando um outro comportamento, ao invés de diretamente pelas suas próprias variáveis controladoras. Em geral, o comportamento diretamente controlado é dito ser governado pela contingência, enquanto o comportamento adjuntivo é dito ser induzido pela contingência. Essa distinção é importante pelo fato do primeiro ser aquele que é programado por meio das relações contingenciais, enquanto o segundo ocorre como um correlato dessas relações.

Os achados iniciais de Falk geraram um grande interesse pelo fenômeno e desencadeou uma variedade de pesquisas na área. Além da polidipsia, outros comportamentos adjuntivos têm sido demonstrados em estudos realizados com animais. Entre esses comportamentos podemos citar, como exemplos, o consumo de álcool em ratos (Lester, 1961), ingestão de materiais não comestíveis (pica) em macacos (Villareal, 1967), agressão em pombos (Hutchinson, Azrin, \& Hunt, 1968), uso da roda de atividades em ratos (Levitsky \& Collier, 1968), lambedura de jatos de ar em ratos (Mendelson \& Chillag, 1970), motilidade intestinal em ratos (Rayfield, Segal \& Goldiamond, 1982), hiperfagia em ratos (Wilson \& Cantor, 1987) e auto-administração de drogas em ratos (Falk, Vigorito, Tang \& Lau, 1990). Além disso, a polidipsia adjuntiva tem sido amplamente utilizada como modelo no estudo dos efeitos produzidos por diferentes substâncias.

Uma série de estudos subsequientes enfatizou os efeitos de determinadas drogas sobre a polidipsia adjuntiva. A esse respeito, Barret (1984) ressaltou o papel dos princípios da Análise Experimental do Comportamento como componente essencial para a pesquisa psicofarmacológica. Segundo o autor, princípios importantes para se compreender o comportamento também são imprescindíveis para a compreensão dos efeitos comportamentais de determinadas drogas.

Snodgrass e Allen (1988) observaram os efeitos da apomorfina, um agente dopaminérgico, sobre a aquisição da polidipsia adjuntiva. Utilizando ratos sob FI $60 \mathrm{~s}$, os autores testaram diferentes doses $(0.05,0.5$ e $1.0 \mathrm{mg} / \mathrm{kg}$ ) e observaram que tanto a polidipsia quanto a resposta de pressão à barra foram diminuídas quando doses altas da droga foram administradas. Para doses baixas, entretanto, somente o padrão de resposta à barra foi afetado. Os autores sugeriram que o perfeito funcionamento do sistema dopaminérgico seria necessário para a aquisição da polidipsia, mas não puderam assegurar que o neurotransmissor esteja especificamente envolvido na produção do beber excessivo.

Mudanças na distribuição temporal da polidipsia adjuntiva foram observadas com a utilização de danfetamina e diazepam (Pellon \& Blackman, 1992). Esses autores observaram que para os três ratos utilizados como sujeitos sob esquema de FI 60s aos quais foram administradas doses de d-anfetamina $(0.25,0.5,1.0$ e $2.0 \mathrm{mg} / \mathrm{kg})$, ocorreu um aumento no beber no início do intervalo entre reforços, no entanto, a droga não provocou um aumento no consumo total de água durante a sessão. Um efeito contrário foi observado quando diazepam $(0.5,1.0,2.0$, e 4.0 $\mathrm{mg} / \mathrm{kg}$ ) foi administrado a outros três sujeitos produzindo uma diminuição no consumo de água no início do intervalo entre reforços que não foi acompanhado de um decréscimo na taxa de polidipsia da sessão. Os mesmos resultados foram obtidos por Flores e Pellon (1997) em estudo sobre os efeitos da d-anfetamina utilizando um esquema múltiplo intervalo fixo e intervalo variável com diferentes durações $(15,30,60$ e 120 s). Os autores notaram que as mudanças no consumo de água ocorreram em função do tamanho do intervalo entre reforços.

O paradigma da polidipsia também vem sendo utilizado para verificar o efeito de novas drogas. Tung, Wu, Tseng e Yin (1994) observaram os efeitos do amperozide, uma droga antipsicótica, sobre a polidipsia. Utilizando ratos como sujeitos, e diferentes doses $(2.0,4.0$ e $8.0 \mathrm{mg} / \mathrm{kg})$, os autores verificaram que cada dose diminuiu significativamente a quantidade de lambidas e do consumo de água. A resposta de pressão à barra sofreu um decréscimo somente quando foram administrados $8.0 \mathrm{mg} / \mathrm{kg}$. Tanto a resposta de pressão à barra quanto o consumo de água retornaram aos níveis de linha de base transcorridos cinco dias após a administração da droga.

Apesar da diversidade de estudos sobre o efeito de diferentes drogas sobre a polidipsia, pouco se sabe sobre os efeitos de outros agentes tóxicos, como a radiação ionizante, sobre comportamentos adjuntivos.

Os estudos sobre os efeitos radiogênicos têm privilegiado aspectos naturalísticos do comportamento bem como alterações biológicas provocadas pela radiação. Apesar do número restrito de pesquisas, os efeitos da radiação ionizante sobre comportamentos sob contingências de reforçamento têm sido avaliados sistematicamente durante a última década. Por outro lado, os possíveis efeitos da radiação ionizante sobre comportamentos adjuntivos ainda não foram investigados. Nesse sentido, o objetivo principal deste trabalho foi realizar uma exploração inicial dos efeitos da radiação ionizante sobre comportamentos adjuntivos, utilizando a polidipsia adjuntiva, o fenômeno protótipo desse tipo de comportamento.

Uma das medidas que tem se mostrado sensível aos efeitos da radiação ionizante é a resposta de pressão à barra. Mudanças na taxa de respostas podem ser obtidas mesmo com pequenas doses de radiação $\left(1,0 \mathrm{~Gy}^{2}\right)$ (Mickley, Bogo, Landauer \& Mele, 1988). Assim, o arranjo experimental utilizado nesse estudo busca analisar os efeitos da radiação ionizante sobre a polidipsia adjuntiva tanto em esquema de reforçamento contingente à resposta (intervalo fixo) quanto em esquema não-contingente à resposta (tempo fixo) permitindo assim uma análise mais abrangente do fenômeno.

\section{MÉTODO}

\section{Sujeitos}


Quatro ratos albinos, machos, experimentalmente ingênuos, com aproximadamente 120 dias de vida no início do experimento, foram utilizados inicialmente como sujeitos experimentais. Esses animais foram divididos aleatoriamente em duas duplas e denominados T1 e T2, I1 e I2. Um quinto animal, I3, de igual procedência, foi posteriormente incorporado ao experimento.

A água disponível livremente na gaiola-viveiro foi fornecida por uma mesma fonte mineral, comercialmente disponível. O controle da qualidade da água tinha por objetivo diminuir os efeitos de possíveis infecções oportunísticas. Além disso, os animais recebiam em média $12 \mathrm{~g}$ de ração Labina / Purina ${ }^{\circledR}$ balanceada para ratos e camundongos ao término da sessão experimental, além do alimento obtido nas sessões experimentais. Todos os animais foram mantidos segundo os princípios enunciados pelo Guide for the Care and Use of Laboratory Animal Research National Research Council, 1996.

\section{Equipamentos}

Para a coleta de dados foi utilizada uma caixa de condicionamento operante construída na oficina do Laboratório de Análise Experimental do Comportamento da Universidade de Brasília, similar à caixa modular para pequenos roedores modelo E10-10 da Coulburn Instruments Inc. O painel de respostas era constituído, da esquerda para direita de uma barra de respostas, um comedouro e um bebedouro. A barra de respostas, medindo $4,0 \times 2,0 \mathrm{~cm}$, ficava localizada a 2,0 $\mathrm{cm}$ da parede lateral e a $8 \mathrm{~cm}$ do assoalho. A abertura do comedouro medindo $4,5 \times 5,5 \mathrm{~cm}$ ficava a $3 \mathrm{~cm}$ da barra. A abertura do bebedouro, medindo 5,0 x $5,0 \mathrm{~cm}$ ficava a $2,5 \mathrm{~cm}$ da abertura do comedouro e a $1,5 \mathrm{~cm}$ da parede lateral da caixa experimental. $\mathrm{O}$ comedouro permanecia fora do alcance do animal durante todo o intervalo programado. Somente durante a ocorrência de reforço o comedouro permitia o acesso à comida em pó do lado de dentro da caixa por um período de três segundos. A iluminação da caixa era feita por uma lâmpada de $6 \mathrm{w}$ colocada no teto junto à parede oposta ao painel de respostas. Durante o acionamento do comedouro, uma outra lâmpada de $6 \mathrm{w}$ garantia a iluminação do mesmo. A caixa de condicionamento era mantida dentro de uma caixa atenuadora de luz e som, com um exaustor que fornecia ventilação e produzia um ruído tênue.

Um equipamento denominado drinkometer fabricado pela Med Associates Inc. (modelo ENV250) registrava as respostas de lamber. Cada vez que o animal lambia o bico da mamadeira uma resposta era registrada através do contato elétrico produzido por um terminal colocado no assoalho da caixa experimental e outro no bico da mamadeira, garantindo a produção de um pulso a cada lambida.

A programação das sessões e os registros das respostas de pressão à barra e das respostas de lambida foram programados por meio do programa Schedule Manager ${ }^{\mathrm{TM}}$ for Windows ${ }^{\circledR}$ produzidos por MED Associates Inc. Um microcomputador executava a programação acoplado a uma interface para controle de processos de fabricação MED Associates Inc. As sessões experimentais foram monitoradas diariamente através de uma câmera de vídeo ligada a um monitor que ficava numa sala adjacente à de coleta.

\section{Procedimentos}

Linha de Base para a resposta de beber - Todos os sujeitos, com exceção de I3, foram submetidos à fase de linha de base na qual o seguinte procedimento foi programado. Durante os primeiros 14 minutos da sessão os sujeitos não tinham acesso a comida. Em seguida os animais tinham acesso a alimento em pó por 3 segundos, com intervalo de 1 segundo, por 30 vezes, com duração total de 2 minutos. Esse período foi denominado reforço maciço e representa o total de acesso ao alimento, quando liberado por 3 segundos a cada minuto, numa sessão de 30 minutos. Após este período, outros 14 minutos eram transcorridos sem que o sujeito tivesse acesso à comida. Todas as sessões experimentais tiveram duração de 30 minutos durante os quais água esteve sempre disponível.

Liberação de Alimento Independente da Resposta - Após 7 sessões na condição de linha de base os sujeitos T1 e T2 foram submetidos a um esquema de tempo FT $60 \mathrm{~s}$ com acesso ao alimento por 3 segundos. Respostas de lambida foram registradas assim como respostas de pressão à barra as quais não tinham consequiência programada.

\section{Liberação Dependente da Resposta}

Modelagem e fortalecimento da resposta de pressão à barra - Os sujeitos I1 e I2, após passarem pelas sessões de linha de base, foram submetidos a um procedimento de modelagem da resposta de pressão à barra. Depois de estabelecida, a resposta de pressão à barra foi reforçada de acordo com o esquema de reforçamento contínuo (CRF), e posteriormente, com quatro valores de esquema de intervalo fixo FI (FI $5 \mathrm{~s}$; FI $10 \mathrm{~s}$; FI $15 \mathrm{~s}$ e FI $30 \mathrm{~s}$ ) em sessões sucessivas. O procedimento de modelagem e treino teve duração de seis sessões tanto para o sujeito I1 como para o sujeito I2. Tanto as respostas de pressão à barra como a de lamber o bico da garrafa de água, foram registradas durante as sessões experimentais. Um novo sujeito, I3, posteriormente incorporado ao experimento também foi exposto a essa fase.

Esquema de reforçamento em Intervalo Fixo Após a aquisição e o fortalecimento da resposta de pressão à barra, os sujeitos experimentais foram submetidos a sessões sob FI $60 \mathrm{~s}$ durante o qual a resposta de pressão à barra era reforçada com acesso ao alimento por 3 segundos segundo a programação acima. Respostas de pressão à barra e de lambida foram registradas durante os 30 minutos da sessão experimental.

\section{Irradiação}

Os sujeitos T1, T2, I1 e I2 foram submetidos à primeira dose de radiação ionizante de 4,5 Gy depois da polidipsia ter sido estabelecida e registrada por aproximadamente 100 sessões. Transcorridos 36 dias da primeira irradiação, os sujeitos T1, T2 e I2 foram submetidos à uma segunda dose de 6,0 Gy de radiação 
ionizante. O sujeito $\mathrm{I} 3$ foi submetido somente a uma dose de 6,0 Gy.

Protocolo de Irradiação - $\mathrm{O}$ protocolo de irradiação foi o mesmo utilizado por Vasconcelos (1999). Os sujeitos foram irradiados em um acelerador linear (Varian, modelo 600/100) utilizado para tratamento médico radioterápico. $\mathrm{O}$ acelerador linear foi calibrado para água como meio espalhador, o que determinou a escolha de um fantoma de água, que possibilitou melhor homogeneização na distribuição das curvas de mesma dose. $\mathrm{O}$ fantoma de água media 15 x 17 x $37 \mathrm{~cm}$, com quatro câmaras de $40 \mathrm{~mm}$ de diâmetro onde os animais eram colocados. A Figura 1 apresenta um esquema representativo do fantoma utilizado.

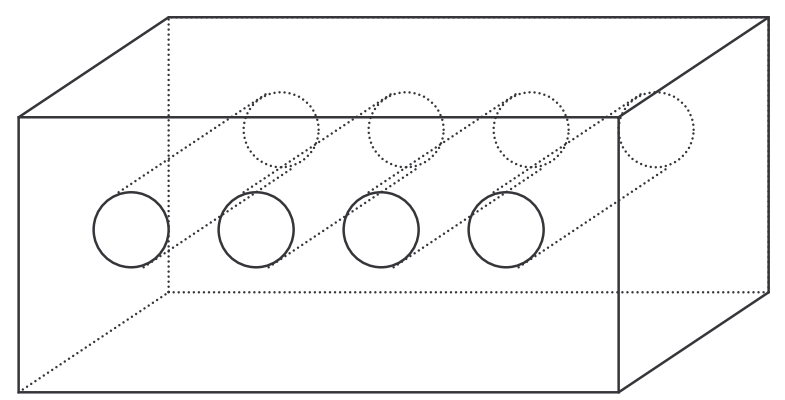

Figura 1 - Esquema representativo do fantoma utilizado no procedimento de irradiação dos ratos.

As sessões de irradiação ocorreram sempre aos domingos. Os sujeitos T1, T2, I1 e I2 foram irradiados dois a dois na primeira sessão com exposição de 4,5 Gy. Na segunda sessão de irradiação (6,0 Gy), participaram os sujeitos T1, T2 e I2 os quais foram irradiados em um único grupo de três. O intervalo entre as duas exposições foi de 36 dias, permitindo assim a recuperação dos efeitos comportamentais e coleta de dados de linha de base para a próxima irradiação. O sujeito I3 foi irradiado $(6,0$ Gy) individualmente.

\section{RESULTADOS}

Os efeitos da radiação ionizante serão descritos e analisados a partir das duas variáveis dependentes do presente estudo: resposta de pressão à barra e respostas de lamber. Além disso, aspectos relacionados ao estabelecimento e à manutenção da polidipsia também serão alvo de descrição e análise.

\section{Estabelecimento da Polidipsia}

\section{$\mathrm{T} 1$}

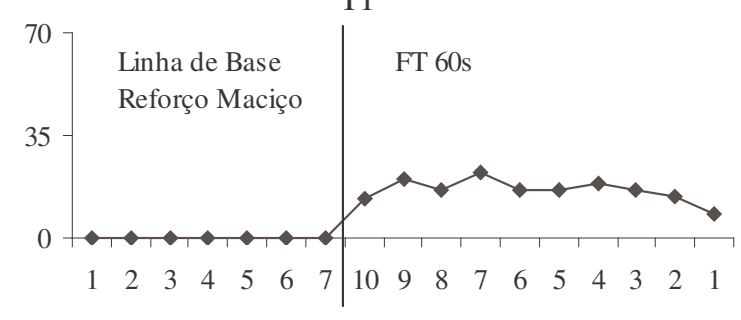

I1

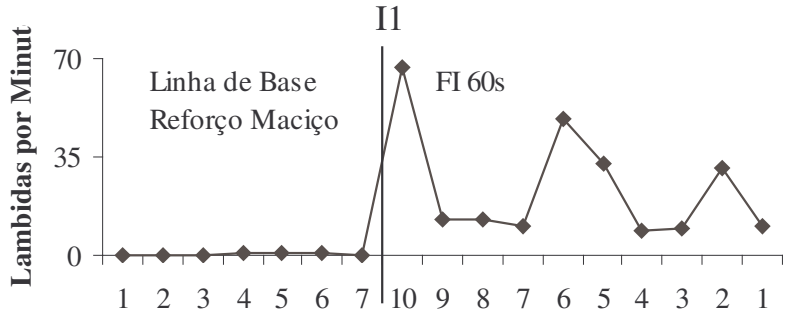

A Figura 2 apresenta a taxa de respostas de lamber (lambidas por minuto) para os sujeitos T1, T2, I1 e I2 nas sete sessões de linha de base e nas últimas dez sessões pré-radiação, e para o sujeito $\mathrm{I} 3 \mathrm{em}$ cinco sessões de linha de base e nas 10 sessões pré-radiação. Para os primeiros sujeitos, nas sessões de linha de base foi utilizada a condição de reforço maciço, descrito anteriormente. Para o sujeito I 3 as sessões de linha de base foram realizadas utilizando esquema de intervalo fixo de 5 segundos (FI $5 \mathrm{~s}$ ). Esse esquema é funcionalmente equivalente ao CRF tendo em vista o comportamento de limpeza gerado pelo alimento em pó. Quando o animal emite a primeira resposta após o reforço, o intervalo geralmente já transcorreu, tornando a probabilidade de reforço dessa resposta próximo a 1 . Nas 10 sessões pré-radiação vigorava o esquema de reforçamento FT $60 \mathrm{~s}$ para os sujeitos T1 e T2 e FI 60 s para os sujeitos I1, I2 e I3. Para todos os sujeitos a taxa de respostas de lamber nas sessões de linha de base foi próxima de zero, enquanto que taxas mais altas de lambida foram registradas nas 10 sessões pré-radiação.

base e nas 10 sessões anteriores à exposição à radiação.
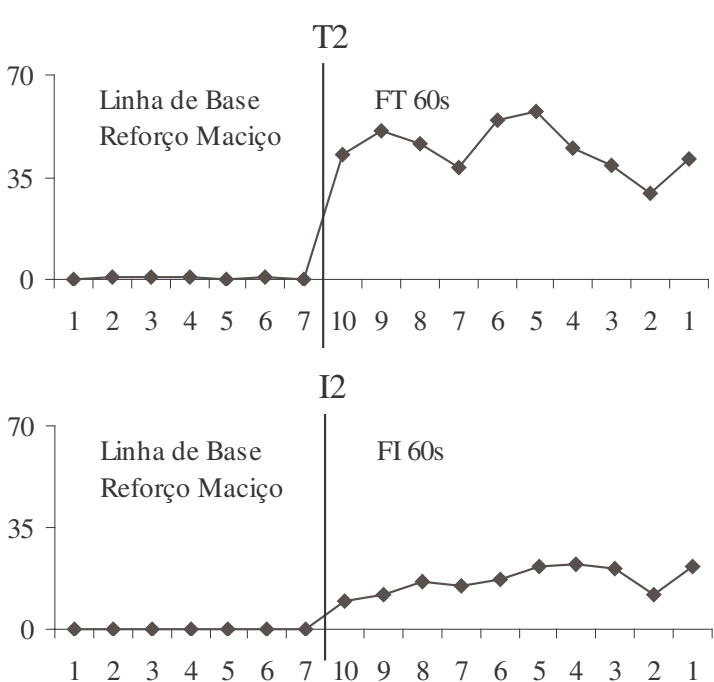


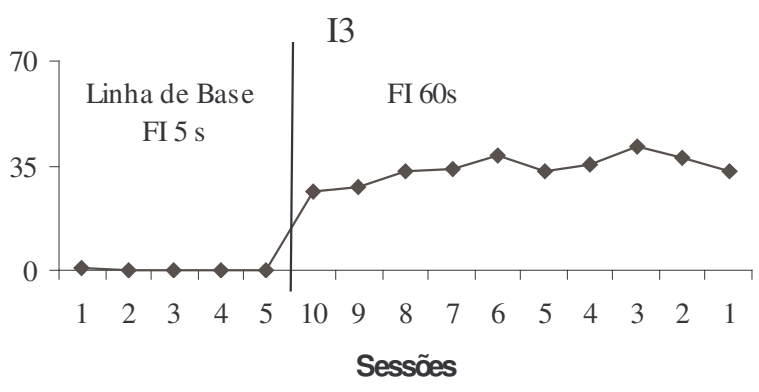

\section{Resposta de Pressão à Barra}

A Figura 2 apresenta a taxa relativa de resposta de pressão à barra em relação à linha de base para as diferentes doses de radiação ionizante $(4,5$ Gy e 6,0 Gy) nas sessões realizadas 2, 24, 48 e 72 horas após a exposição, para os sujeitos I2, I1 e I3. O sujeito I2 apresentou uma queda maior na taxa relativa de resposta na sessão realizada 2 horas após a exposição para a dose 4,5 Gy enquanto que se observou um efeito maior para a dose 6,0 Gy nas sessões realizadas 48 e 72 horas pós-radiação, alcançando valores relativos próximos a zero. Taxas relativas de respostas semelhantes foram registradas para as duas doses $(4,5$ Gy e 6,0 Gy) na segunda sessão (24 horas) pósradiação. $\mathrm{O}$ sujeito $\mathrm{I} 1$ mostrou queda na taxa relativa de resposta em relação à linha de base nas quatro sessões após a exposição à radiação ionizante de 4,5 Gy, com um maior efeito podendo ser observado na sessão que ocorreu 72 horas pós-radiação. O sujeito I3 foi exposto somente à dose de radiação de 6,0 Gy e intensificação dos efeitos sobre a taxa relativa de resposta na sessão realizada 48 horas após a exposição. A ausência dos dados do sujeito I1 para a dose de 6,0 Gy se deveu à morte do sujeito após a primeira exposição à radiação devido a infecções decorrentes da fragilidade do sistema imunológico facilitadas por problemas no equipamento de ventilação e filtragem de ar do biotério. Para o sujeito I3 não foi programada outra exposição que aquela realizada $(6,0 \mathrm{~Gy})$. 
I2

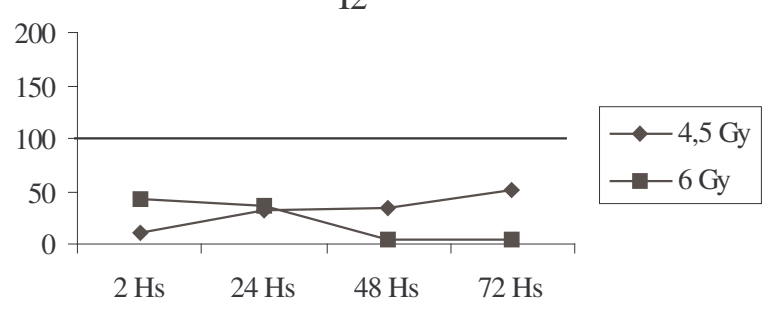

I1

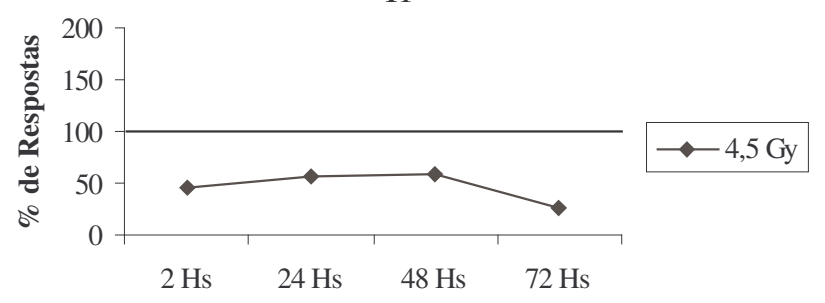

I3

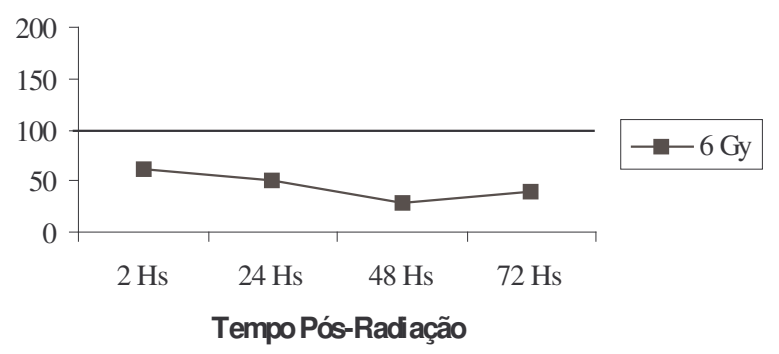

Figura 3 - Taxa relativa de respostas de pressão à barra em relação à linha de base para os sujeitos 12 e I1 e I3 nas quatro sessões que se seguiram à exposição à radiação ionizante. A linha de base (100\%) corresponde à média da taxa de respostas nas 10 últimas sessões pré-radiação.

\section{Resposta de Lamber}

A Figura 3 mostra os efeitos das duas doses de radiação ionizante na taxa relativa de lambidas nas sessões realizadas 2, 24, 48 e 72 horas após a exposição para os sujeitos T1, T2, I2 e de uma dose para os sujeitos I1 e I3. Os dados mostram que a dose de 4,5 Gy provocou uma queda mais acentuada na porcentagem de lambidas para os sujeitos T2, I2 e I1 na sessão realizada 2 horas após a exposição, enquanto que para a dose 6,0 Gy esse efeito foi observado na terceira sessão (48 horas) pós-radiação para T1, T2, I2 e I3. Todos os sujeitos expostos à dose
4,5 Gy mostraram recuperação da taxa de lambida aos níveis de linha de base na sessão realizada 72 horas após a exposição, enquanto que somente T1 mostrou a mesma recuperação para a dose de 6,0 Gy. Além disso, o sujeito $\mathrm{T} 1$ registrou valores relativos de lambida muito superiores à linha de base após a exposição de 4,5 Gy. Com a dose de 6,0 Gy pode também ser observado uma maior redução nas taxas, para os sujeitos expostos aos esquemas de FI, em relação aos sujeitos expostos ao esquema de FT, 48 horas após a exposição à radiação. $\mathrm{ré}^{\prime}$

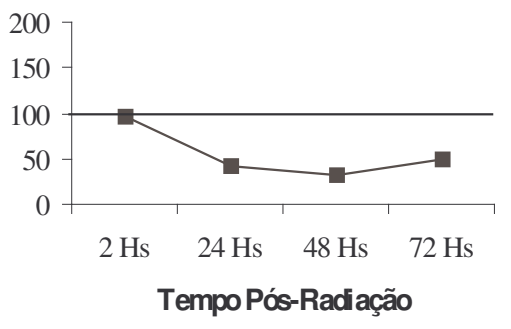

I2 (FI 60s)

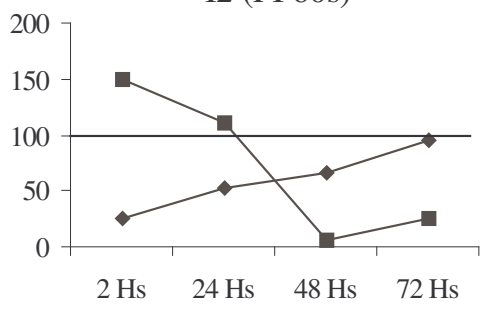

à

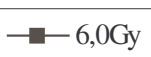




\section{DISCUSSÃO}

\section{Efeitos da Radiação Ionizante sobre a resposta de pressão à barra}

A resposta de pressão à barra tem sido alvo de diversos estudos por ter se mostrado particularmente sensível aos efeitos da radiação ionizante. A medida de taxa de resposta tem apresentado alterações mesmo utilizando-se doses baixas de radiação. Tais efeitos podem ser observados em diversos procedimentos: em esquemas simples de intervalo fixo (Gimenes, Vasconcelos \& Goulart, 1991), em esquemas simples de intervalo variável (Vasconcelos, Gimenes, Magalhães, Santos, Macedo, Brandão \& Goulart, 1993), em esquemas concorrente de reforçamento (Vasconcelos, Gimenes, Brandão, Bezerra, Dacanal, Campos \& Goulart, 1995), em esquemas múltiplos de razão fixa e intervalo fixo (Mele \& McDounough, 1995), em aquisição repetida (Winsauer \& Mele, 1993), e em esquema múltiplo de aquisição repetida e desempenho (Vasconcelos, 1999).

Os resultados obtidos no presente estudo apontam para uma diminuição na taxa de respostas para os sujeitos expostos à radiação ionizante com dose de 4,5 Gy. Esse efeito se mostrou intensificado na sessão realizada duas horas após a exposição, com posterior recuperação da taxa de resposta sem, entretanto, alcançarem os níveis de linha de base. Resultado semelhante foi encontrado por Gimenes e cols. (1991) que observaram efeitos sobre o desempenho em FI mesmo em doses mais baixas, de 3,5 Gy. Além disso, esses autores observaram um efeito seletivo no que se refere às taxas de resposta na condição de linha de base. Sujeitos que responderam com uma taxa mais alta durante a linha de base, apresentaram uma maior redução na taxa de respostas após a irradiação.

O desempenho dos sujeitos I2 e I3 após a exposição à radiação ionizante com dose de 6,0 Gy no presente experimento demonstra esse efeito seletivo. $\mathrm{O}$ sujeito que respondeu com taxas mais altas durante a condição de linha de base demonstrou uma maior queda do que aquele que respondeu com taxas mais baixas. Para essa dose, a intensificação dos efeitos da radiação ionizante foi pronunciada na sessão realizada 48 horas após a exposição. Entretanto, observou-se recuperação de desempenho próximo à linha de base a partir da quinta sessão pós-radiação. Esses resultados sugerem que assim como o esquema de reforçamento, a taxa de resposta na condição de linha de base desempenha papel fundamental na modulação dos efeitos da radiação ionizante. Resultados semelhantes foram obtidos por Mele e cols. (1988), utilizando esquema de FR 50 e FI 120 s. As altas taxas produzidas pelo esquema FR 50 sofreram maior queda após a exposição de 6,5 Gy que aquelas produzidas pelo esquema de FI 120 s. Além disso, observaram outro efeito no que tange ao padrão típico do esquema, ou seja, para o FI $120 \mathrm{~s}$ a queda na taxa de respostas não foi acompanhada de uma alteração no padrão típico do esquema, enquanto que para o esquema FR 50 a alteração foi marcante no que se refere às pausas pós-reforço. As reduções nas taxas de resposta foram acompanhadas por aumento das pausas pós-reforço. De forma geral os dados do presente experimento, em relação aos efeitos da radiação ionizante sobre a resposta de pressão à barra, replicam dados encontrados na literatura. Os padrões observados apresentaram redução nas taxas, principalmente durante o período de 2 a 72 horas após a irradiação e recuperação gradual das mesmas. Além disso, os efeitos foram dose-dependentes, com a maior dose produzindo um maior efeito, ou seja, produzindo uma redução mais intensa nas taxas de resposta.

As quedas nas taxas de respostas no presente estudo também foram acompanhadas de quedas nas taxas de reforços após a exposição à radiação ionizante. Embora tenha havido essa redução, a mesma não foi da mesma intensidade daquela observada para as taxas de respostas. Somente quando as taxas de respostas mostraram reduções mais acentuadas, pode-se observar reduções marcantes nas taxas de reforços. Esses efeitos também foram dosedependentes, ou seja, a taxa de reforços sofreu maior decréscimo quando da exposição à radiação com dose de 6,0 Gy se comparada à dose de 4,5 Gy. Essas observações também corroboram dados previamente apresentados (Vasconcelos e cols., 1995).

Uma explicação para a redução na taxa de respostas e na taxa de reforços após exposição à radiação foi proposta por Winsauer, Verrees, O'Halloran, Bixler e Mele (1994). Segundo os autores, os efeitos anoréxicos da radiação levariam à uma redução no consumo de alimento, sendo que a duração desse efeito está diretamente relacionada com a dose a qual o animal foi exposto, ou seja, quanto maior a dose, maior o efeito anoréxico provocado por ela, levando à uma redução na taxa de respostas. Além disso, a queda na atividade geral, acompanhada de letargia também parece determinar a queda observada na taxa de respostas, o que estaria diretamente associada à redução na taxa de reforços e conseqüente redução no consumo dos mesmos. Embora esses efeitos anoréxicos e letárgicos possam ser claramente observados em situação livre, isto é, nas gaiolas viveiro por exemplo, os mesmos tendem a ser modulados por contingências operantes. Em trabalhos anteriores Gimenes e Vasconcelos (1999) demonstraram esse tipo de modulação, com diferentes tipos de contingências afetando taxas de respostas, mas não taxas de reforços obtidos ou consumo dos mesmos. A diferença entre os efeitos sobre as taxas de respostas e taxas de reforços obtidos no presente estudo também aponta para essa mediação das contingências operantes sobre os efeitos da radiação ionizante.

\section{Efeitos da Radiação Ionizante sobre a Polidipsia}

Efeitos comportamentais radiogênicos semelhantes àqueles encontrados sobre a taxa de respostas foram observados sobre a taxa de lamber no presente estudo. Após a exposição à radiação ionizante de 4,5 Gy, três dos quatro sujeitos sofreram queda na taxa de lambidas na primeira sessão (2 horas), realizada após 
a exposição com posterior recuperação próxima à linha de base. Da mesma forma, não foram observadas diferenças sistemáticas nos efeitos da radiação na taxa de respostas de lamber em relação aos esquemas de tempo fixo (FT 60 s) e intervalo fixo (FI $60 \mathrm{~s}$ ).

Por outro lado, um efeito diferenciado foi observado quando da exposição a 6,0 Gy. As taxas de lambidas após a exposição a 6,0 Gy mostraram queda mais acentuada do que após a exposição à dose de 4,5 Gy. Além disso, os dados do presente estudo apontam para um maior decréscimo da taxa de respostas de lamber para aqueles sujeitos sob esquema de intervalo fixo se comparado a aqueles em tempo fixo. Os sujeitos em intervalo fixo mostraram uma queda na taxa relativa de lambidas superior a $50 \%$, chegando próxima a zero para o sujeito I2 na terceira sessão pós-radiação, diferentemente dos sujeitos em tempo fixo, cuja diminuição da taxa relativa de lambidas foi inferior a $50 \%$.

Assim, os resultados do presente estudo sugerem que existe uma interação entre o comportamento operante e o comportamento adjuntivo que pode modular os efeitos da radiação ionizante. Entretanto, essa modulação não foi observada quando da exposição à doses mais baixas de radiação, sugerindo assim que existe um limiar de dose onde os efeitos da radiação sobre o comportamento operante interagem diretamente com os efeitos provocados sobre o comportamento adjuntivo. Os efeitos letárgicos produzidos pela radiação ionizante podem também ter contribuído para as diferenças observadas entre os sujeitos expostos aos esquemas em FT e FI. Os esforços necessários para as respostas de pressão à barra podem ter interferido com a resposta de beber uma vez que a mesma requer custos adicionais característicos da topografia exigida pelo equipamento bem como deslocamento adicional dentro da caixa experimental.

Estudos realizados sobre efeitos de drogas sobre a polidipsia encontraram resultados semelhantes a aqueles apresentados no presente trabalho. Snodgrass e Allen (1988) estudaram os efeitos da apomorfina, um agente dopaminérgico sobre o estabelecimento da polidipsia adjuntiva com diferentes doses e observaram que tanto a polidipsia quanto a resposta de pressão à barra foram diminuídas quando doses altas da droga foram administradas. Para doses baixas, entretanto, somente o padrão de respostas à barra foi afetado. Essa semelhança nos resultados sugere que o sistema dopaminérgico pode ser um dos moduladores dos efeitos comportamentais produzidos pela radiação ionizante. De fato, Foulon, Lalouette, Lambert, Martin, Fatome e Martin (1999) observaram em ratos que a exposição à radiação ionizante ocasionou uma diminuição dos níveis de dopamina e de seus metabólitos.

Da mesma forma que o sistema dopaminérgico pode estar modulando os efeitos da radiação ionizante sobre a polidipsia, podem também estar modulando os efeitos sobre a resposta operante de pressão à barra. Cory-Slechta, Pazmino e Bare (1997) demonstraram também que reduções nos níveis de dopamina são seguidas de reduções nas taxas de respostas em esquema de reforçamento de intervalo fixo. Estudos posteriores deverão ser realizados no sentido de investigar a participação do sistema dopaminérgico na modulação dos efeitos da radiação ionizante sobre a resposta operante de pressão à barra bem como sobre a polidipsia induzida por esquema de reforçamento.

De uma maneira geral, os resultados produzidos pelo presente trabalho buscaram explorar aspectos relacionados aos efeitos da radiação ionizante sobre a polidipsia até então não estudados. Estudos posteriores serão necessários no sentido de ampliar o conhecimento acerca das variáveis que interagem no estabelecimento e na manutenção da polidipsia bem como das variáveis relacionadas aos efeitos comportamentais da radiação ionizante. Os efeitos diferenciados observados nos sujeitos expostos aos diferentes esquemas indutores, FT e FI, sinalizam uma interação entre o comportamento operante e o comportamento adjuntivo no que se refere aos efeitos radiogênicos. Estudos que avaliem essa interação, bem como a generalidade do fenômeno, podem ajudar a clarificar as variáveis responsáveis pelo mesmo como também suas interações com a radiação ionizante.

Quanto à variável radiação ionizante, a utilização cada vez maior da energia nuclear torna necessário que estudos sejam realizados no sentido de identificar e analisar os efeitos biológicos e comportamentais de diferentes variáveis radiológicas fornecendo à comunidade estratégias que possam diminuir os danos provocados pela exposição à radiação ionizante.

\section{REFERÊNCIAS}

Barrett, J. E. (1984). Behavioral principles in psychopharmacology. Em D. J. Sanger \& D. E. Blackman (Orgs.), Psychology in Progress. Aspects of Psychopharmacology (pp. 20-56). London: Methuen.

Cory-Slechta, D. A.; Pazmino, R. \& Bare, C. (1997). The critical role of nucleus accumbens dopamine system in the mediation of fixed interval schedule-controlled operant behavior. Brain Research, 764 (1-2), 253-256.

Falk, J. L. (1961a). Production of polydipsia in normal rats by an intermittent food schedule. Science, 133, 195-196.

Falk, J. L. (1961b). The behavioral regulation of water-electrolyte balance. Em M. R. Jones (Org.), Nebraska Symposium on Motivation. Lincoln: University of Nebraska Press, 1-33.

Falk, J. L. (1971). The nature and determinants of adjunctive behavior. Physiology and Behavior, 6 (5), 577-588.

Falk, J. L.; Vigorito, M.; Tang, M. \& Lau, C. E. (1990). Scheduleinduced cocaine drinking: Choice between cocaine and vehicle. Pharmacology, Biochemistry \& Behavior, 35, 187193.

Flores, P. \& Pellón, R. (1997). Effects of d-amphetamine on temporal distributions of schedule-induced polydipsia. Pharmacology, Biochemistry and Behavior, 57 (1-2), 81-87.

Foulon, O.; Lalouette, F.; Lambert, F.; Martin, S.; Fatome, M. \& Martin, C. (1999). Effects of neutron-gamma radiation on dopamine and serotonin metabolism in the rat brain: a regional analysis. Journal of Neuroscience Research, 55 (6), 770-775.

Gimenes, L. S. \& Vasconcelos, L. A. (1999). Efeitos da radiação ionizante sobre comportamentos mantidos por contingências operantes. Psicologia: Teoria e Pesquisa, 15 (3), 219-225.

Gimenes, L. S.; Vasconcelos, L. A. \& Goulart, F. B. (1991) Effects of ionizing radiation on operant performance by rats under interval schedules of reinforcement. Em Association for 
Behavior Analysis (Org.), Proceedings of the $17^{\text {th }}$ Annual Convention of the Association for Behavior Analysis (p. 264). Atlanta, USA: ABA.

Hutchinson, R. R.; Azrin, N. H. \& Hunt, G. M. (1968). Attack produced by intermittent reinforcement of a concurrent operant response. Journal of the Experimental Analysis of Behavior, 11, 489-495.

Lester, D. (1961). Self-maintenance of intoxication in the rat. Quarterly Journal of Studies on Alcohol, 22, 223-231.

Levitsky, D. \& Collier, G. (1968). Schedule-induced wheel running. Physiology and Behavior, 3, 571-573.

Mele, P. C. \& McDonough, J. H. (1995). Gamma radiationinduced disruption in schedule-controlled performance in rats. Neurotoxicology, 16, 497-510.

Mendelson, J. \& Chillag, D. (1970). Schedule-induced air licking in rats. Physiology and Behavior, 5, 535-537.

Mickley, G. A.; Bogo, V.; Landauer, M. R. \& Mele, P. C. (1988). Current trends in behavioral radiobiology. Em P. D McCormack; C. E. Swenberg \& H. Bucker (Orgs.), Terrestrial Space Radiation and its Biological Effects (pp. 517-536). New York: Plenum Publishing Company.

National Research Council (1996). Guide for the Care and Use of Laboratory Animals. Washington: National Academy Press.

Pellon, R. \& Blackman, D. E. (1992). Effects of drugs on the temporal distribution of schedule-induced polydipsia in rats. Pharmacology, Biochemistry and Behavior, 43 (3), 689-695.

Rayfield, F.; Segal, M. \& Goldiamond, I. (1982). Scheduleinduced defecation. Journal of the Experimental Analysis of Behavior, 38, 19-34.

Snodgrass, S. H. \& Allen, J. D. (1988). The effects of apomorphine on the acquisition of schedule-induced polydipsia in rats. Pharmacology, Biochemistry and Behavior, 29 (3), 483-488.

Tung, C. S.; Wu, W. H.; Tseng, C. J. \& Yin, T. H. (1994). Effects of amperozide on schedule-induced polydipsia in rats. European Journal of Pharmacology, 256 (2), 193-200.

Vasconcelos L. A.; Gimenes, L. S.; Brandão, A. M.; Bezerra, A. C. A.; Dacanal, J. N.; Campos, L. M. \& Goulart, F. B. (1995). Irradiação ionizante e o desempenho de ratos sob esquema concorrente VI-VI. Em Sociedade Brasileira de Psicologia (Org.), Resumos de Comunicações Científicas da XXV Reunião Anual de Psicologia (p. 444). Ribeirão Preto: SBP.

Vasconcelos, L. A. (1999). Radiação Ionizante: Efeitos sobre a Aquisição e o Desempenho do Comportamento Operante. Tese de Doutorado. Universidade de Brasília, Brasília.

Vasconcelos, L. A.; Gimenes, L. S.; Magalhães, E. R.; Santos, A C. D.; Macedo, J. L.; Brandão, A. \& Goulart, F. B. (1993) Efeitos de doses subletais de irradiação ionizante sobre o desempenho em intervalo variável em ratos. Em Sociedade Brasileira de Psicologia (Org.), Resumos de Comunicações Científicas da XXIII Reunião Anual de Psicologia (p. 484). Ribeirão Preto, SBP.

Villareal, J. (1967, maio). Schedule-induced "pica". Trabalho apresentado no Annual Meeting of the Eastern Psychological Association, Boston, USA.

Wilson, J. F. \& Cantor, M. B. (1987). An animal model of excessive eating: Schedule-induced hyperphagia in foodsatiated rats. Journal of the Experimental Analysis of Behavior, 47, 335-346.

Winsauer, P. J. \& Mele, P. C. (1993). Effects of sublethal doses of ionizing radiation on repeated acquisition in rats. Pharmacology. Biochemistry and Behavior, 44, 809-814.

Winsauer, P. J.; Verrees, J. F.; O'Halloran, K. P.; Bixler, M. A. \& Mele, P. C. (1994). Effects of chlordizepoxide, 8-OH-DPAT and ondansetron on radiation-induced decreases in food intake in rats. The Journal of Pharmacology and Experimental Therapeutics, 270, 142-149. 


\begin{abstract}
Notas:
${ }^{1}$ Apoio Financeiro: $\mathrm{CNPq}$

${ }^{2}$ Gray (Gy) é a unidade de dose absorvida no Sistema Internacional. A dose absorvida é definida como sendo a energia absorvida da radiação pela unidade de massa.
\end{abstract}

\title{
Sobre os autores:
}

Alessandra de Moura Brandão: Mestra em Psicologia (Processos Comportamentais) e Doutoranda em Psicologia pela Universidade de Brasília. Bolsista do CNPq. E-mail: $\underline{\text { abrandao@unb.br }}$

Lincoln da Silva Gimenes: Ph.D. em Ciências do Comportamento (Psicobiologia) pela University of Chicago. Professor do Departamento de Processos Psicológicos Básicos do Instituto de Psicologia da Universidade de Brasília. E-mail: lgimenes@unb.br

Júlio Luiz Rodrigues: Mestre em Física Nuclear e Planejamento Energético pela Universidade Federal do Rio de Janeiro. Físico Médico do Hospital Santa Lúcia. Email: julio.bsb@brturbo.com

\section{Endereço para correspondência:}

Universidade de Brasília - Depto. de Processos Psicológicos Básicos

Instituto de Psicologia - Campus Universitário Darcy Ribeiro

70.910-900 Brasília - DF 